\title{
Medición radiográfica del ángulo acetabular para el diagnóstico temprano de la displasia del desarrollo de la cadera
}

\author{
Osorio-García JJ,* Mendieta-Alcántara GG** \\ Hospital Materno Perinatal «Mónica Pretelini Sáenz»
}

RESUMEN. Antecedentes: Para evitar secuelas en la displasia del desarrollo de la cadera el tratamiento debe ser temprano; el ángulo acetabular corresponde a la inclinación del techo del acetábulo. Stanisavljevic indica que para medir adecuadamente el ángulo acetabular se deben colocar las caderas en abducción. Pregunta: ¿cuál es la magnitud de la diferencia de la medición radiográfica del ángulo acetabular entre las proyecciones anteroposterior de pelvis en posición neutra y con abducción de caderas? Métodos: Se tomaron 916 mediciones radiográficas comparando las de posición neutra contra las de abducción de caderas, de pacientes atendidos en consulta externa entre el 1 de Abril de 2010 y el 31 de Marzo de 2013. Resultados: Se realizaron pruebas t para medias de dos muestras emparejadas y se obtuvo una diferencia hipotética de las medias de 0.0000 y el coeficiente de correlación de Pearson para cadera derecha fue 0.74427531 y para la izquierda 0.73779866 . Usando una tabla propuesta de normalidad del ángulo acetabular según edad, se obtuvo el área bajo la curva en una distribución $\mathrm{t}_{\mathrm{gl} 457}=0.650$ con $\mathrm{p}=\mathbf{0 . 5 1 6}$. El índice acetabular en posición neutra y con abducción de caderas es similar con alta significancia estadística, según el análisis de correlación de Pearson. Con base en la curva ROC, el índice acetabular en posición de rana tiene una alta sensibilidad y especificidad. Por tanto, la medición del índice acetabular en posición con abducción de caderas, tiene alta confiabilidad de lograr un diagnóstico correcto.

Palabras clave: Displasia, cadera, ángulo acetabular, radiografía, diagnóstico.
ABSTRACT. Background: In order to avoid sequelae in the development dysplasia of the hip the treatment should be early; The acetabular angle corresponds to the inclination of the roof of the acetabulum. Stanisvljevic indicates that to properly measure the acetabular angle, the hips should be placed in abduction. Question: What is the magnitude of the difference in radiographic measurement of the acetabular angle between the anteroposterior projections of pelvis in neutral position and with abduction of hips? Methods: 916 radiographic measurements were taken by contrasting the neutral position against those of hip abduction, of patients in our clinic attended between April 1, 2010 and March 31, 2013. Results: $T$ tests were performed for means of two matched samples and a hypothetical difference of the mean of 0.0000 was obtained and the Pearson correlation coefficient for right hip was $\mathbf{0 . 7 4 4 2 7 5 3 1}$

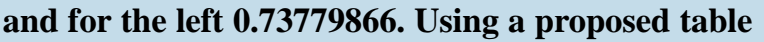
of normality of the Acetabular angle according to age, the area was obtained under the curve in a distribution $T_{\text {gl457 }}=0.650$ with $P=0.516$. The index acetabular in neutral position and with abduction of hips is similar with high statistical significance, according to the analysis of correlation of Pearson. Based on the ROC curve, the acetabular index in frog position has a high sensitivity and specificity. Discussion: Therefore, the measurement of the acetabular index in position with hip abduction, has high reliability to achieve a correct diagnosis.

Key words: Dysplasia, hip, acetabular angle, $\mathrm{x}$-ray, diagnosis.

Nivel de evidencia: IV

* Médico Cirujano Especialista en Ortopedia. Servicio de Cirugía Ortopédica.

** Doctor en Ciencias de la Salud, Servicio de Cardiología Pediátrica.

Hospital Materno Perinatal «Mónica Pretelini Sáenz» del Instituto de Salud del Estado de México, Toluca.

Dirección para correspondencia:

Jesús Javier Osorio García

Instituto Literario Poniente Núm. 502-9, Col. Centro, CP 50000, Toluca, Estado de México. Teléfono: (722) 215 67 54

E-mail: jjosoriogarcia@gmail.com

Este artículo puede ser consultado en versión completa en http://www.medigraphic.com/actaortopedica 


\section{Introducción}

La displasia del desarrollo de la cadera (DDC), anteriormente conocida como luxación congénita de la cadera, integra anormalidades anatómicas que afectan la articulación coxofemoral del niño(a) incluyendo el borde anormal del acetábulo (displasia) y mala posición de la cabeza femoral, causando desde subluxación hasta luxación, afectando el desarrollo de la cadera durante los períodos embriológico, fetal o infantil. . $, 2,3,4^{2}$

Los doctores Alfredo Iñárritu Cervantes y Pedro Antonio Bravo Bernabé en el Programa de Actualización Continua para Médicos Generales, en el capítulo correspondiente, señalan que la luxación congénita de la cadera, también llamada displasia luxante, miodisplasia congénita o malformación luxante, es una malformación congénita cuyo reconocimiento reviste mucha importancia, no sólo por su frecuencia, sino porque de no descubrirse tempranamente, puede producir un grave deterioro de la articulación afectada, con la consiguiente pérdida de la función y la invalidez que ello significa. En la inestabilidad articular, la acción del tono y de la contracción muscular con los movimientos del niño pequeño tienden a desplazar la cabeza femoral en sentido proximal. ${ }^{5} \mathrm{El}$ doctor Alvarado Brown señala que la displasia evolutiva de cadera es una de las enfermedades congénitas de mayor prevalencia a nivel mundial. Según Klisic «esta enfermedad en realidad indica un desorden dinámico, potencialmente capaz, en la medida que el bebé se desarrolla, de mejorar o empeorar» ${ }^{6}$

El reconocimiento precoz de una cadera displásica permite un tratamiento temprano, simple, efectivo y libre de complicaciones. ${ }^{7}$ Pero cuando este diagnóstico se hace tardío las consecuencias de una articulación mal desarrollada no pueden, en muchos de los casos, ser solucionadas por las múltiples técnicas de tratamiento existentes y el resultado es una alteración anatómica que muy pronto en el adolescente o en el adulto necesitan procedimientos reconstructivos como osteotomías pélvicas, osteotomías femorales o finalmente reemplazos articulares que imponen un altísimo costo social, humano y económico. Por lo tanto, todos los esfuerzos que se puedan realizar para establecer un sistema objetivo y viable de diagnóstico temprano de la displasia del desarrollo de la cadera están justificados ${ }^{5,8}$ La verdadera solución de este padecimiento estriba en el diagnóstico temprano en el recién nacido o antes del año de edad, cuando el tratamiento temprano conduce habitualmente a la curación del padecimiento., ${ }^{9,10,11}$

Finalmente y aceptando que la displasia de la cadera es una entidad de naturaleza genética, que no siempre está presente al nacimiento y que se puede desarrollar posterior al período neonatal, se acepta la importancia de no sólo un cuidadoso examen físico en el recién nacido, sino de su repetición periódica y juiciosa durante el primer año de vida; además de siempre tener presente la «identificación del recién nacido de alto riesgo», utilizando los factores de riesgo como una guía en los programas de tamizaje clínico, radiológico o ecográfico. ${ }^{8,12,13,14}$
El mayor rendimiento de los estudios radiográficos se logran después de los dos meses de vida (algunos autores hablan del cuarto a sexto mes), ya que en la DDC los primeros cambios sobre las estructuras óseas ocurren después de las cuatro a seis semanas de manifestada la laxitud articular. En la radiografía de pelvis para el estudio de DDC deben realizarse algunas mediciones básicas. Se traza la línea de Hilgenreiner horizontalmente uniendo los extremos inferiores del íleon o los extremos superiores de las ramas isquiopubianas. Se traza una línea a lo largo del techo del acetábulo y que corte la línea de Hilgenreiner. El ángulo entre ambas es el índice acetabular. Este ángulo se considera anormal por encima de $30^{\circ}$. A partir del año de vida debe ser menor de $25^{\circ}$. Por último se dibujan unas líneas verticales que pasen por el margen lateral del acetábulo. El núcleo de osificación de la cabeza femoral debe estar en el cuadrante inferior y medial. Es de gran importancia, no sólo para hacer el diagnóstico, sino para hacer la valoración de las alteraciones existentes que van a condicionar el tratamiento y apreciar la mejoría que el tratamiento va logrando en el transcurso del crecimiento del enfermo.

La radiografía de la pelvis del recién nacido muestra una imagen muy especial por encontrarse sus núcleos de osificación del isquion, el ilion y el pubis separados por el cartílago trirradiado que no es visible. La cabeza femoral aparece en la radiografía a los cinco o seis meses de edad, apreciándose mientras tanto solamente osificado el muñón trocantérico. Por estas razones, la interpretación es difícil si no se localizan puntos de referencia constantes que, relacionados entre sí, permitan evaluar el estudio:

El fondo acetabular correspondiente al sitio en donde confluyen los tres elementos del cartílago trirradiado. El punto más externo del acetábulo visible que corresponde al borde acetabular. El centro del núcleo de osificación de la cabeza femoral, cuando es visible. La porción más alta del muñón diafisario cuando aún no aparece la cabeza femoral.

Los trazos geométricos que los relacionan son: la línea de Hilgenreiner, horizontal que une el fondo acetabular de ambos lados. El punto más alto de la cabeza, o en su defecto el muñón trocantéreo, debe encontrarse de 0 a $4 \mathrm{~mm}$ por abajo de la línea de Hilgenreiner. Su elevación por encima de ella significa luxada. La línea trazada desde el fondo acetabular hasta el borde acetabular, que forma un ángulo abierto hacia afuera, y se denomina ángulo o índice acetabular, mide la inclinación del techo acetabular. La distancia entre el fondo acetabular y la parte más próxima de la cabeza femoral o del muñón trocantéreo debe ser normalmente de 5 a $11 \mathrm{~mm}$. Una distancia mayor significa ocupación del acetábulo por elementos que no permiten la penetración de la cabeza, subluxándola o descentrándola.

El ángulo o índice acetabular anatómico corresponde a la inclinación del techo del acetábulo y, por tanto, da la medida del grado de displasia, que es la capacidad de recubrimiento y de contención sobre la cabeza. Su valor ha sido fijado en $30^{\circ}$ máximo para el recién nacido por múltiples autores. En 1961, Sharp demostró que la basculación de la pelvis y/o su 
oblicuidad al momento de la toma de las radiografías modifican la medición del ángulo acetabular. ${ }^{15}$

Fijar en $30^{\circ}$ el máximo normal en menores de tres meses en radiografía con piernas extendidas es adecuado para los doctores Sierra y Fernández. ${ }^{16}$ A partir de las seis semanas de vida se pueden apreciar los cambios radiográficos en el acetábulo, así como el desplazamiento lateral de la cabeza y de la metáfisis femoral. La radiografía de cadera debe tomarse con la cadera en posición neutra. La proyección de Von Rosen consiste en colocar las piernas en ángulo de 45 grados, en abducción y con los muslos internamente rotados; acentuando la dislocación de la cadera que puede no ser aparente en las proyecciones de rutina. ${ }^{17,18}$ Las proyecciones radiográficas de la cadera que se recomiendan para el estudio de los niños con DDC son en posición neutra y la proyección de rana. ${ }^{1}$

Stanisavljevic indica que para medir adecuadamente el índice acetabular anatómico en el recién nacido se debe colocar la pelvis con el sacro aplanado en la mesa y las piernas flexionadas y en abducción. Si las piernas se extienden a esta edad, la pelvis se bascula al frente y entonces aparece en el techo del acetábulo parte del área posterior del mismo, lo que erróneamente mejora la medida. Stanisavljevic ha calculado que tiene en el recién nacido un valor promedio de $28^{\circ}{ }^{16}$

La displasia del desarrollo de la cadera es una patología muy común en nuestro medio, es la causa más frecuente de consulta en el Servicio de Cirugía Ortopédica de nuestro hospital. La sospecha de esta patología se basa en la exploración que realizan los médicos neonatólogos al valorar a los recién nacidos y posteriormente son remitidos a valoración por nuestro servicio. A todos los pacientes que son remitidos por sospecha de patología de las caderas se les solicitan radiografías de pelvis para medir el ángulo acetabular, la literatura señala hacer la medición del ángulo en la proyección neutra; ${ }^{19}$ sin embargo, Stanisavljevic asegura que la mejor proyección radiográfica para la medición de la inclinación del techo acetabular es la proyección con abducción de las caderas (mejor conocida como posición de rana) debido a que es más exacta.

Con base en la recomendación de Stanisavljevic, ${ }^{20,21}$ en nuestro servicio se realizó la medición del ángulo acetabular en ambas proyecciones radiográficas (en posición neutra y en posición de rana).

\section{Material y métodos}

Se realizó un estudio observacional, retroprospectivo y analítico al contrastar las mediciones radiográficas del índice acetabular de los pacientes, obtenidas en dos diferentes proyecciones, en posición neutra y con abducción de las caderas.

Este estudio se llevó a cabo con la autorización del Comité de Enseñanza e Investigación y el Comité de Ética en Investigación del hospital bajo las siguientes implicaciones: el manejo de la información obtenida de los expedientes clínicos fue bajo la más estricta confidencialidad. Debido a que los expedientes clínicos que se utilizaron para este estudio son propiedad intelectual del hospital y a que no se revelarán datos individuales de los pacientes incluidos en el estudio no se requiere autorización escrita de los pacientes para integrar sus datos al proceso estadístico.

Se revisaron 1,396 notas médicas de igual número de consultas otorgadas en la Consulta Externa del Servicio de Cirugía Ortopédica a pacientes que fueron atendidos entre el 1 de Abril de 2010 y el 31 de Marzo de 2013, 229 consultas cumplieron con los criterios, lo que representó 458 caderas y 916 mediciones radiográficas de ángulo acetabular realizadas. A cada paciente le fueron tomadas radiografías de pelvis en proyección anteroposterior en posición neutra y en posición de rana, por lo que cada cadera de los pacientes fue medida en las dos posiciones ya descritas. Se utilizó estadística descriptiva, calculando medidas de tendencia central y de dispersión básicamente en cuanto a medias y desviaciones estándar de los ángulos acetabulares en ambas proyecciones radiográficas y ambas caderas. Para contrastar las medias de los índices acetabulares de las caderas en posición neutra y posición de rana se utilizó una prueba de t pareada, escogiendo esta prueba ya que ambas mediciones se hicieron en cada uno de los pacientes. Se calculó el coeficiente de correlación de Pearson entre ambas proyecciones. Se realizó una curva ROC tomando como estándar de oro la posición neutra para identificar sensibilidad y especificidad de la medición en la posición de rana.

Con base en la literatura consultada se desarrolló la $T a-$ bla 1 para calificar cada cadera evaluada en función de

Tabla 1: Normalidad del ángulo acetabular según la edad.

$\begin{array}{ccc}\text { Edad en meses } & \text { Normal } & \text { Máximo } \\ 1 & 30 & 30 \\ 2 & 29 & 30 \\ 3 & 28 & 29 \\ 4 & 27 & 29 \\ 5 & 26 & 28 \\ 6 & 25 & 28 \\ 7 & 24 & 27 \\ 8 & 23 & 27 \\ 9 & 22 & 27 \\ 10 & 22 & 26 \\ 11 & 21 & 26 \\ 12 & 20 & 25 \\ 13 & 19 & 25 \\ 14 & 18 & 25 \\ 15 & 17 & 24 \\ 16 & 16 & 24 \\ 17 & 15 & 24 \\ 18 & 15 & 23 \\ 19 & 14 & 23 \\ 20 & 13 & 22 \\ 21 & 12 & 22 \\ 22 & 11 & 21 \\ 23 & 10 & 21 \\ 24 & 10 & 20 \\ & & \end{array}$


la medición del ángulo acetabular en grados y de la edad cronológica en meses de cada paciente. De tal manera que si la cadera presentaba un valor superior al límite máximo señalado según la edad del paciente fue calificada como displasia; en caso contrario, cuando la cadera arrojaba una medición del ángulo acetabular menor o igual al límite máximo establecido según su edad en meses entonces se le calificó como normal. Las consideraciones anteriores en cuanto a la Tabla 1 se utilizaron para el diseño de la curva ROC.

Se tomaron los valores del ángulo acetabular en la $\mathrm{Ta}$ bla 1 considerando la posición neutra de acuerdo con lo señalado por múltiples autores. Se procesaron los datos en una hoja de cálculo con el programa Excel de Office versión 2010, ordenando la información por fecha de atención, registrando la fecha de nacimiento y la fecha de la toma de las radiografías para obtener la edad automáticamente al momento de la medición, se usó este mismo programa para la calcular la correlación de Pearson y también se utilizó para el cálculo de la prueba de t pareada. Para el cálculo y diseño de la curva ROC se utilizó el programa SPSS versión 20.0.

\section{Resultados}

Al realizar la contrastación de medias para ambas caderas, en la cadera derecha encontramos para la posición neutra una media de $25.99^{\circ} \pm 5.77^{\circ}$ y para la posición de rana de $25.75^{\circ} \pm 5.24^{\circ}$, se calculó entonces la t pareada con un resultado de una $\mathrm{t}_{\mathrm{g} 1228}=1.98$ con una $\mathrm{p}=0.38$. En la cadera izquierda en posición neutra se encontró una media de $24.15^{\circ} \pm 5.15^{\circ}$ y para la posición de rana resultó una media de $24.15^{\circ} \pm 4.89^{\circ}$, los resultados para la cadera izquierda son de $\mathrm{t}_{\mathrm{g} 1228}=1.97$ con una $\mathrm{p}=1.0$. La correlación de Pearson tuvo como resultado un coeficiente de 0.748 con una significancia $<0.001$.

En la Figura 1 se observa la curva ROC, la cual resultó con un área bajo dicha curva de 0.841 con un error típico de 0.026 e intervalo de confianza a $95 \%$ que abarca un área de 0.791 como límite inferior a 0.892 como límite superior y una $\mathrm{p}<0.001$ tomando como área bajo la curva a contrastar en la hipótesis nula de 0.5 .

Las medias son similares porque no existen diferencias estadísticamente significativas por la prueba de $t$ pareada. Las mediciones del índice acetabular radiográfico en las proyecciones en posición neutra y con abducción de caderas tienen una correlación altamente significativa con base en el análisis de correlación de Pearson.

Tomando en cuenta la medición del índice acetabular radiográfico en posición neutra como estándar de oro, entonces con base en el análisis de la curva ROC se considera que la medición del índice acetabular radiográfico en posición con abducción de caderas es una prueba que tiene una alta sensibilidad y especificidad.

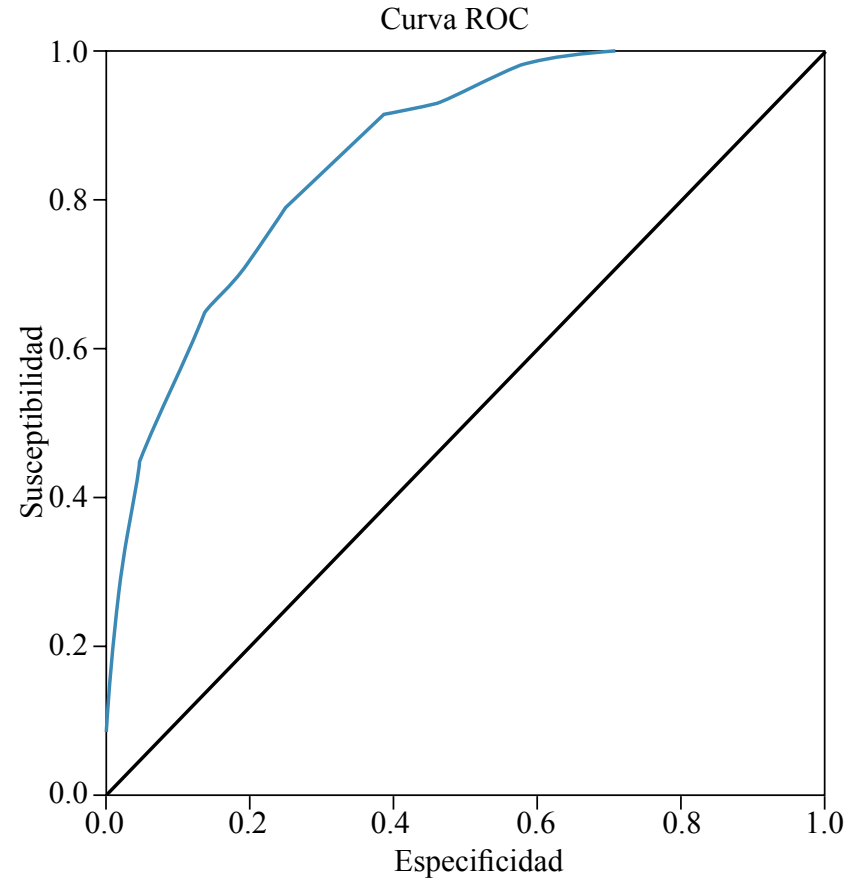

Los segmentos diagonales son producidos por empates

Figura 1: Curva ROC.

\section{Discusión}

La medición radiográfica del ángulo acetabular en la proyección anteroposterior de pelvis en posición con abducción de caderas tiene, con una alta significancia estadística, el mismo valor diagnóstico como la medición radiográfica en la posición neutra en los pacientes con sospecha de displasia del desarrollo de la cadera.

Cuando se carece de la proyección radiográfica en posición neutra y se cuenta con la medición de los índices acetabulares en la proyección en posición con abducción de caderas, existe una alta confiabilidad de lograr un diagnóstico correcto con sólo ésta última proyección radiográfica.

Stanisavljevic asegura que la proyección radiográfica en posición con abducción de las caderas (mejor conocida como posición de rana) es más exacta; con base en este estudio, encontramos que la medición del índice acetabular en proyección de rana es similar estadísticamente a la tomada en posición neutra y para efectos de diagnóstico es muy cercana en especificidad y sensibilidad.

Bibliografía

1. Diagnóstico y tratamiento oportuno de la displasia en el desarrollo de cadera. México: Secretaría de Salud; 2008.

2. Murillo-Quiroga M, Zegarra-Mita H, Castellón-Tamez JL. Displasia de cadera en desarrollo. Rev Paceña Med Fam. 2008; 5(8): 88-91.

3. http://es.wikipedia.org/wiki/Displasia [Consultada el 10/05/12].

4. http://www.portalesmedicos.com/diccionario_medico/index.php/ Displasia [Consultada el 10/05/12]. 
5. http://www.drscope.com/privados/pac/generales/pdl1/cadera.html [Consultada el 10/05/12].

6. Alvarado-Brown A. Displasia evolutiva de cadera (DEC). Rev Med Cos Cen. 2009; 66(588): 155-9.

7. Dezateux C, Brown J, Arthur R, Karnon J, Parnaby A. Performance, treatment pathways, and effects of alternative policy options for screening for developmental dysplasia of the hip in the United Kingdom. Arch Dis Child. 2003; 88(9): 753-9.

8. http://www.encolombia.com/orto12198programa.htm [Consultada el 10/05/12].

9. Elbourne D, Dezateux C. Hip dysplasia and ultrasound imaging of whole populations: the precautionary principle revisited. Arch Dis Child Fetal Neonatal Ed. 2005; 90(1): F2-3.

10. Harcke HT. The role of ultrasound in diagnosis and management of developmental dysplasia of the hip. Pediatr Radiol. 1995; 25(3): 225-7.

11. Lowry CA, Donoghue VB, Murphy JF. Auditing hip ultrasound screening of infants at increased risk of developmental dysplasia of the hip. Arch Dis Child. 2005; 90(6): 579-81.

12. Macnicol MF. Results of a 25-year screening programme for neonatal hip instability. J Bone Joint Surg Br. 1990; 72(6): 1057-60.

13. Monge-Bonilla C, Sánchez-Ramírez Adriana, Morales-Alpízar C, Serrano-Aybar P. Diagnóstico tardío de displasia evolutiva de cadera en la población infantil costarricense en el período 1996-2000. Acta Med Costarric. 2002; 44(3): 117-20.

14. Arce VJD, García BC. Displasia del desarrollo de caderas: ¿radiografía o ultrasonografía? ¿A quiénes y cuándo? Rev Chil Pediatr. 2000; 71(4): 354-6.

15. Sharp IK. Acetabular dysplasia: the acetabular angle. $J$ Bone Joint Surg Br. 1961; 43: 268-72.

16. Sierra-Rojas L, Fernández Herrera E. Luxación congénita de la cadera. México: Limusa; 1992.

17. Tachdjian M. Ortopedia pediátrica. Buenos Aires: Editorial Interamericana; 1994, 322-564.

18. Terver SP, Constine RM, Csongradi J, Kleinman R, Bleck EE. Congenital dislocation of the hip--prognostic implications of early diagnosis. West J Med. 1979; 131(2): 98-103.

19. Sánchez Ruiz-Cabello J. Cribado de la displasia evolutiva de cadera. Revista Prev Infad. 2002; 30: 28-34.

20. Stanisavljevic S. Diagnosis and treatment of congenital hip pathology in the newborn. Baltimore: Williams \& Wilkins Co.; 1964, 94.

21. Stanisavljevic S, Mitchell CL. Congenital dysplasia, subluxation, and dislocation of the hip in stillborn and newborn infants. $J$ Bone Joint Surg Am. 1963; 45: 1147-58. 